OPEN ACCESS

Edited by:

Anders Sjöstedt,

Umeå University, Sweden

Reviewed by:

Richard Titball,

University of Exeter, United Kingdom

Siouxsie Wiles,

University of Auckland, New Zealand

*Correspondence:

Johanna Thelaus johanna.thelaus@foi.se

Received: 06 March 2018 Accepted: 15 May 2018

Published: 05 June 2018

Citation:

Thelaus J, Lundmark E, Lindgren P, Sjödin A and Forsman M (2018) Galleria mellonella Reveals Niche

Differences Between Highly

Pathogenic and Closely Related

Strains of Francisella spp.

Front. Cell. Infect. Microbiol. 8:188.

doi: 10.3389/fcimb.2018.00188

\section{Galleria mellonella Reveals Niche Differences Between Highly Pathogenic and Closely Related Strains of Francisella spp.}

\author{
Johanna Thelaus*, Eva Lundmark, Petter Lindgren, Andreas Sjödin and Mats Forsman \\ Swedish Defence Research Agency (FOl), Umeå, Sweden
}

Francisella tularensis, a highly virulent bacteria that causes the zoonotic disease tularemia, is considered a potential agent of biological warfare and bioterrorism. Although the host range for several species within the Francisella is known, little is known about the natural reservoirs of various Francisella species. The lack of knowledge regarding the environmental fates of these pathogens greatly reduces the possibilities for microbial risk assessments. The greater wax moth (Galleria mellonella) is an insect of the order Lepidoptera that has been used as an alternative model to study microbial infection during recent years. The aim of this study was to evaluate G. mellonella as a model system for studies of human pathogenic and closely related opportunistic and non-pathogenic strains within the Francisella genus. The employed G. mellonella larvae model demonstrated differences in lethality between human pathogenic and human non-pathogenic or opportunistic Francisella species. The F. novicida, F. hispaniensis and $F$. philomiragia strains were significantly more virulent in the G. mellonella model than the strains of human pathogens F. t. holarctica and F. t. tularensis. Our data show that G. mellonella is a possible in vivo model of insect immunity for studies of both opportunistic and virulent lineages of Francisella spp., that produces inverse results regarding lethality in $G$. mellonella and incapacitating disease in humans. The results provide insight into the potential host specificity of $F$. tularensis and closely related members of the same genus, thus increasing our present understanding of Francisella spp. ecology.

Keywords: tularemia, Francisella tularensis, Galleria mellonella, virulence, host specificity, lethality, ecology

\section{INTRODUCTION}

Francisella tularensis is a highly virulent bacteria that causes the zoonotic disease tularemia. This pathogen is considered a potential agent of biological warfare and bioterrorism. As such, it is classified as a Tier 1 select agent (Federal Register, 2012). Three subspecies of F. tularensis are commonly accepted: F. t. holarctica, F. t. tularensis and F. t. mediasiatica (Sjöstedt, 2015). Both F. tularensis subspecies holarctica and subspecies tularensis cause disease in humans (Penn, 2010). However, information on the virulence of $F$. t. mediasiatica, which is primarily found in Central Asia, in humans is limited (Timofeev et al., 2017). Besides F. tularensis, the genus Francisella also includes opportunistic pathogens that only cause disease in immunocompromised humans (i.e., 
F. novicida, F. hispaniensis and F. philomiragia; Larson et al., 1955; Olsufjev et al., 1959; Hollis et al., 1989; Huber et al., 2010; Penn, 2010; Aravena-Román et al., 2015). There is an ongoing debate about whether F. novicida should be classified as a separate Francisella species or as a subspecies of F. tularensis (Busse et al., 2010; Johansson et al., 2010). The facts that F. novicida infections are uncommon in humans and that infections have only occurred in immunocompromised individuals or people with underlying health problems reflect that $F$. novicida is indeed an opportunistic pathogen (Kingry and Petersen, 2014). In addition, the genus Francisella includes fish pathogens (i.e., F. noatunensis, F. halioticida) (Ottem et al., 2008; Brevik et al., 2011), endosymbionts of ticks and ciliates (i.e., F. persica and F. endociliophora) (Sjödin et al., 2014; Larson et al., 2016), and species with unknown niche preferences, i.e., species isolated from brackish water, air conditioning systems and cooling towers (F. salina, F. uliginis, F. frigiditurris, and Allofrancisella spp.; Qu et al., 2013, 2016; Challacombe et al., 2017). Thus, representatives of the Francisella genus inhabit diverse ecological niches. Rapid developments in high-throughput sequencing technologies during the last years have increased knowledge on the diversity of Francisella and its genetic neighbors (Sjödin et al., 2012; Challacombe et al., 2017).

Although previous research has identified the host preferences of several Francisella species, little is known about the natural reservoirs of these different Francisella species. This lack of knowledge concerning environmental dynamics greatly reduces the possibilities for microbial risk assessments of Francisella pathogens However, the increase in genus diversity knowledge is pivotal to discriminating pathogenic from non-pathogenic strains. This could improve environmental bio-surveillance and epidemiological studies that rely on complex sample matrices which are plagued by false positive signals originating from both non-pathogenic Francisella species and close genetic neighbors. Still, studies of these newly defined organisms are challenged by the difficulties in culturing these strains in the laboratory.

The greater wax moth (Galleria mellonella) is an insect of the order Lepidoptera that has been introduced as an alternative model to study microbial infection during recent years (Tsai et al., 2016). When compared to traditional murine models, G. mellonella larvae are cheaper and easier to maintain. Furthermore, G. mellonella can survive temperatures between 25 and $37^{\circ} \mathrm{C}$, which enables researchers to investigate the hostassociated replication of human pathogens. Although there are major differences between the immune systems of humans and insects, the innate immune responses of insects and vertebrates share a cellular and a humoral component (Kavanagh and Reeves, 2004; Browne et al., 2013). G. mellonella has been used as an infection model to study bacterial and fungal infections, as well as to evaluate the efficacy of antimicrobial substances (Johnson et al., 2015; Tsai et al., 2016; Barnoy et al., 2017; Meir et al., 2018) Additionally, a G. mellonella infection model has been established for the F. $t$. holarctica live vaccine strain (LVS) (Aperis et al., 2007).

The aim of this study was to evaluate whether G. mellonella can be used as a model system to differentiate human pathogenic strains from closely related opportunistic and non-pathogenic
TABLE 1 | Francisella strains included in the study.

\begin{tabular}{lcl}
\hline Strain & FSC number & \multicolumn{1}{c}{ References } \\
\hline F. t. holarctica LVS & 458 & $\begin{array}{l}\text { Isolated from vaccine ampoule, no NDBR } \\
\text { 101, Pasturella tularensis Vaccine Live, lot } \\
\text { no 11, 1964, The National Drug Company, } \\
\end{array}$ \\
& 200 & $\begin{array}{l}\text { Philadelphia, USA } \\
\text { Svensson et al., 2012 }\end{array}$ \\
F. t. holarctica & 237 & Larsson et al., 2005 \\
F. t. tularensis SchuS4 & 147 & Larsson et al., 2009 \\
F. t. mediasiatica & 040 & Larson et al., 1955; Rohmer et al., 2007 \\
F. novicida & 454 & Huber et al., 2010 \\
F. hispaniensis & 037 & Sjödin et al., 2012 \\
F. philomiragia & 1006 & Sjödin et al., 2014 \\
F. endociliophora & &
\end{tabular}

strains within the Francisella genus. We show unexpected differences in lethality between human-pathogenic and human non-pathogenic or opportunistic Francisella species in the G. mellonella larvae model. Thus, the results demonstrate the importance of including non-pathogenic genetic neighbors when evaluating new model systems and suggest niche differences between highly pathogenic and closely related strains of Francisella.

\section{MATERIALS AND METHODS \\ Bacterial Strains Growth Conditions}

All Francisella strains used in this study (Table 1) were cultured on GCII agar containing 1\% hemoglobin and $1 \%$ IsoVitaleX (World Health Organization, 2007), and complemented with $50 \mu \mathrm{g} / \mathrm{mL}$ ampicillin, $100 \mu \mathrm{g} / \mathrm{mL}$ polymyxin B and $25 \mu \mathrm{g} / \mathrm{mL}$ vancomycin. The cultures were incubated at $37^{\circ} \mathrm{C}\left(5 \% \mathrm{CO}_{2}\right)$, except for $F$. endociliophora, which was incubated at $22^{\circ} \mathrm{C}$. Laboratory work with FSC200, FSC147, and FSC237 were performed at a Biosafety level 3 (BSL3) laboratory.

\section{Preparation of Inocula for Infections}

Bacteria were suspended in PBS solution to a final count of 1 $\times 10^{9}$ bacteria/mL as determined by optical density $\left(\mathrm{OD}_{600}\right)$ measurements, and further diluted to give inocula of $10^{8}, 10^{6}$, and $10^{4}$ bacteria/mL. The actual infectious dose was confirmed by plating serial dilutions for an analysis of colony forming units (CFU).

\section{Bacterial Growth Experiments}

Bacteria were suspended in PBS solution to a final count of $10^{5}$ bacteria/mL. Growth rate experiments were performed on solid agar plates where one $\mu l$ of bacterial suspension were applicated in eight technical replicates. Growth was monitored at 18, 32, and $48 \mathrm{~h}$ and scored when bacterial colonies were visible to the eye.

\section{Infection of G. mellonella}

G. mellonella larvae in the final larval stage (Vivara/CJ Wildbird Foods Ltd., Shrewsbury, UK) were stored in the dark at $14^{\circ} \mathrm{C}$. To assess the differences between bacterial concentrations, $10 \mu \mathrm{l}$ of the different dilutions described above were injected into the 
hemocoel of larvae via the last left proleg. After injection, the larvae were incubated in Petri dishes at $37^{\circ}$ or $22^{\circ} \mathrm{C}$ for 9 days. Ten randomly chosen larvae from each group were used to evaluate virulence, and the number of dead larvae was scored every day. Larvae were considered dead when they did not turn after being turned onto their back. All experiments were repeated three times with different batches of larvae. Untreated larvae and larvae injected with PBS were used as controls in all experiments. Upon death or at the end of the experiment, hemolymph was collected from larvae into $1.5 \mathrm{ml}$ Eppendorf tubes for immediate analysis of viable counts or stored at $-80^{\circ} \mathrm{C}$ for confirmative real-time PCR analysis.

\section{Confirmative Culture and Real-Time PCR}

To evaluate the tissue burden of Francisella spp. (for FSC200, FSC040, FSC454, and FSC037) in G. mellonella, the hemolymph collected from infected larvae was serial diluted and plated onto agar plates as described above. In order to confirm that larval death was caused by Francisella sp., all hemolymph samples were screened for the presence of Francisella specific DNA using realtime PCR with the primers Fra_ISFtu2_F CCCTGATTTACA AGAAGTC and Fra_ISFtu2_R CTTGGTTATCATCTTTATCAT ATC and probe Fra_ISFtu2 TGATTCAACAATAGCAAGAGC ACAT (for FSC 458, 200, 237, 147, and 040) modified from Versage et al. (2003), and the primers GF1_F AACTGGCTGACC TTCAGCAT and GF1_R GTGGTCGTGGTAAAGCTGGT and GF1 probe CCGATTAGGCTTTCTGCTACTTCACGA (Sjödin, unpublished) (for FSC 037, 454, and 1006). No DNA extraction was performed prior to the PCR, however, all samples were diluted 1/10 in water to avoid inhibition of the PCR process.

Each reaction mixture comprised $1 \mu \mathrm{l}$ diluted larval hemolymph, 12.5 $\mathrm{ll}$ PerfeCTa qPCR ToughMix (Quantabio, Beverly, MA), $0.625 \mu \mathrm{l}$ of each primer $(20 \mathrm{pM}), 0.5 \mu \mathrm{l}$ of probe (5 pM) and MiliQ water to a total volume of $25 \mu \mathrm{l}$. An initial denaturation at $98^{\circ} \mathrm{C}$ for $2 \mathrm{~min}$ was followed by 45 cycles of $98^{\circ} \mathrm{C}$ for $5 \mathrm{~s}$ and $60^{\circ} \mathrm{C}$ for $5 \mathrm{~s}$ on an iCycler (Bio-Rad, Hercules, CA). Larval hemolymph samples spiked with F. t. holarctica LVS concentrations ranging from $10^{4}$ to $10^{9} \mathrm{CFU}$ per $\mathrm{mL}$ were analyzed in duplicates to test the limit of detection and generate a standard curve for assessing target DNA concentrations. The standard curve was evaluated with FRA_ISFtu2 pimerpair and probe and the GF1 primerpair and probe.

\section{Statistical Analysis}

The Kaplan-Meier estimator was used to estimate the survival function of $G$. mellonella larvae. An event was defined as a dead larva meeting the requirement of a positive PCR measurement (ct $<35,97$ (GF1) and ct $<29,94$ (Fra_ISFtu2), corresponding to $10^{5} \mathrm{cfu} / \mathrm{ml}$. Observations of dead larvae with a negative PCR measurement (ct > 35,97 (GF1) and ct > 29,94 (Fra_ISFtu2)) as well as observations of larvae that had started pupation were considered censored at the time of death and the time of pupation, respectively. The log rank test was used to compare survival functions between groups (i.e., different Francisella species). Analyses for each infectious dose at $37^{\circ} \mathrm{C}$ as well as for the infectious dose of $10^{6}$ bacteria $/ \mathrm{mL}$ at $22^{\circ} \mathrm{C}$ were performed. In addition, we tested the difference in survival functions between strains at $37^{\circ} \mathrm{C}$ when controlled for the infectious dose effect. The control group was omitted from this analysis due to discrepancy in infectious dose. In each analysis, multiple adjustments were performed using the Tukey-Kramer method, with adjusted $p$-values $<0.05$ considered statistically significant. The survival analysis was performed using the PROC LIFETEST in SAS $\left.^{(}\right)$version 9.4 (SAS Institute, Cary, NC). For details see experimental data and sourcecode deposited at figshare (DOI code: 10.6084/m9.figshare.6154919 and DOI data: 10.6084/m9.figshare.6154928).

\section{Phylogenetic Study of Francisella Species}

Sequence files for all publicly available Francisella genomes were downloaded from NCBI (Jan 26, 2018). All of the sequences were aligned using progressiveMauve (Darling et al., 2010), and the multiple alignment was transformed to a common coordinate system (Lärkeryd et al., 2014) before it was imported into MEGA7 (Kumar et al., 2016) to construct a neighborjoining tree. All analysis steps except tree generation were performed using snakemake (Köster and Rahmann, 2012) and Bioconda.

\section{RESULTS}

\section{G. mellonella Mortality Depends on Both Francisella Species and Temperature}

Infection of Galleria mellonella with Francisella strains resulted in larval death (Figure 1). We infected G. mellonella with samples containing $10^{4}, 10^{6}$ or $10^{8}$ bacteria/mL, resulting in actual infectious doses of $10^{2}, 10^{4}$ and $10^{6}$ bacteria per larva (low, intermediate and high dose, respectively. see Figures 1A-C).

At a temperature of $37^{\circ} \mathrm{C}, \mathrm{G}$. mellonella larvae were generally more sensitive to the opportunistic F. novicida, F. hispaniensis, and $F$. philomiragia strains than the other tested strains (Figure 1). This difference was found to be significant $(p<$ 0.001 for all such pairwise combinations) when controlling for infectious dose (Supplementary Table 1, column E). The highest infectious doses of the opportunistic strains resulted in extensive larval death within 3 days while the majority of larvae died within 6-9 days after being injected with the highest infectious doses of the F. tularensis strains (tularensis, holarctica, and mediasiatica). The survival proportion of larvae infected with the highest infectious dose of F. endociliophora was approximately 0.6 at the end of the experiment (Figure 1C), while larvae infected with the lowest and intermediate infectious doses were not affected. At an intermediate infectious dose, wild-type F. $t$. holarctica was significantly more virulent than the F. $t$. holarctica LVS (Figure 1B, Supplementary Table 1 column B). This difference was not observed for the lowest or highest infectious dose (Figures 1A,C, Supplementary Table 1 column A and C).

Francisella infection of $G$. mellonella was also temperaturedependent, as higher larval survival proportions were noted when the experiment was performed at $22^{\circ} \mathrm{C}$ (Figure 2) than when the experiment was performed at $37^{\circ} \mathrm{C}$ (Figure 1). This trend was noted for all strains at the intermediate infectious dose. 

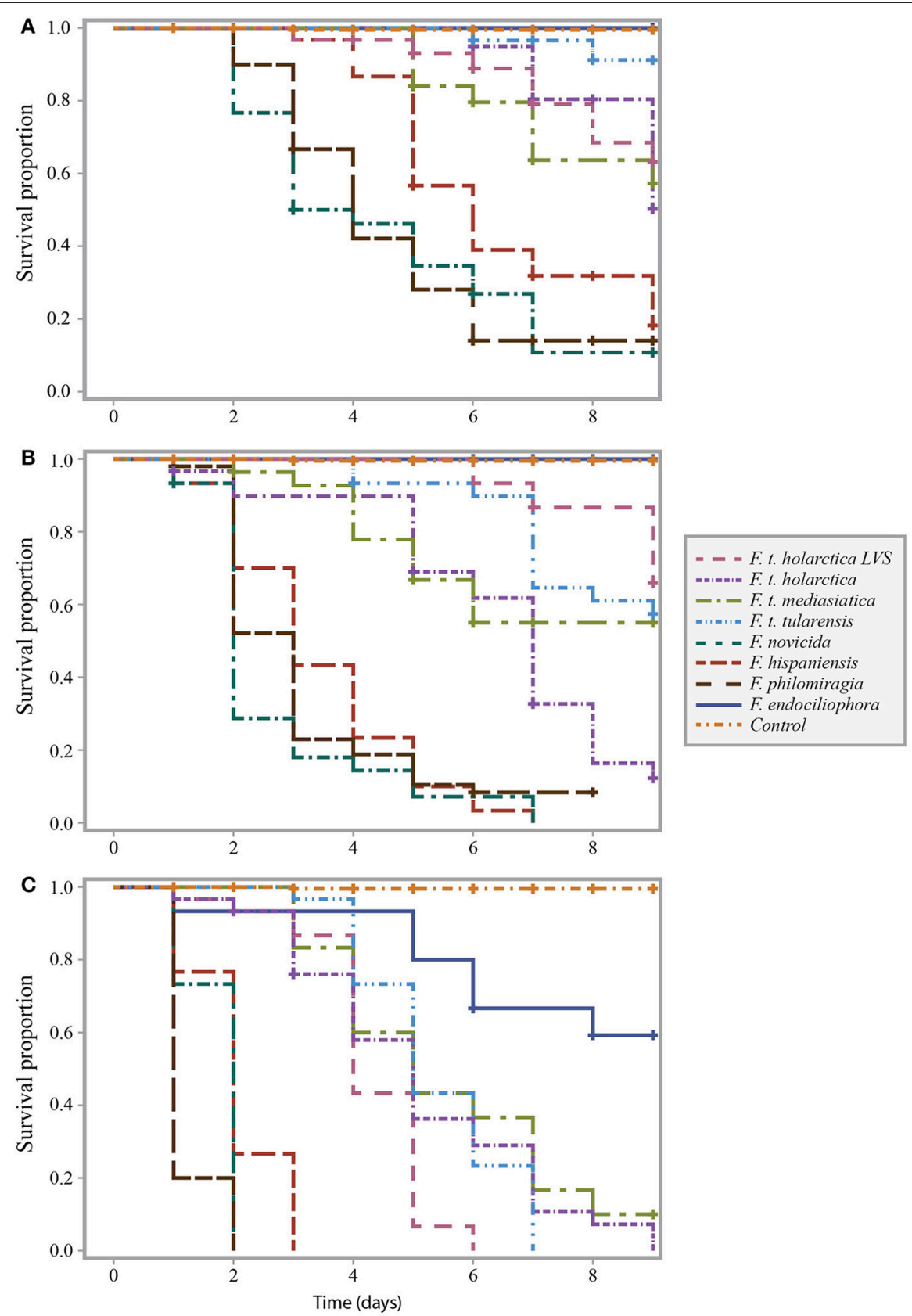

FIGURE 1 | Survival curve comparison of G. mellonella infected with F. t. tularensis, F. t. holarctica, F. t. mediasiatica, F. novicida, F. hispaniensis, F. philomiragia, and F. endociliophora at a temperature of $37^{\circ} \mathrm{C}$ with an infectious dose of (A) $10^{4}$ bacteria/mL, (B) $10^{6}$ bacteria/mL, and (C) $10^{8}$ bacteria/mL. Experiments were performed in three separate runs with ten larvae in each run for each treatment. Censored observations are indicated with a plus sign (+).

\section{Francisella spp. Infect G. mellonella With a Small Infectious Dose and Grow to High Bacterial Count}

The bacterial load of Francisella spp. in G. mellonella hemolymph upon larval death reached approximately $10^{10} \mathrm{CFU} / \mathrm{mL}$ for F. t. holarctica, F. novicida and F. hispaniensis (Figure 3). Based on the assumption that each larvae contains $35 \mu \mathrm{L}$ of hemolymph, the initial $10^{4}$ bacteria introduced upon infection (intermediate dose) reached $3.5 \times 10^{8}$ bacteria per larva at the time of larval death. However, the bacterial load of $F$. philomiragia in hemolymph upon larval death was noticeably lower than what was observed for other Francisella species, not exceeding $10^{8} \mathrm{CFU} / \mathrm{mL}$, which corresponds to $3.5 \times 10^{6}$ bacteria per larva at the time of death (Figure 3).

Taken together, these results suggest that the bacterial load increased by $10^{4}$ over 2 days in samples infected with F. novicida 


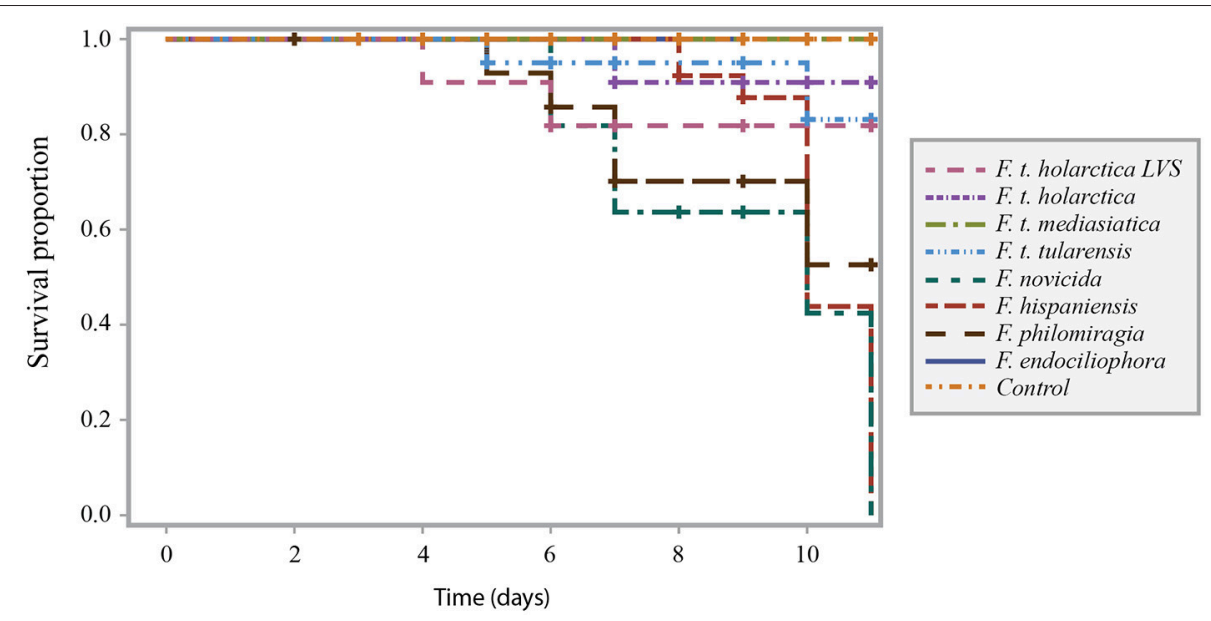

FIGURE 2 | Survival curve comparison of G. mellonella infected with F. t. tularensis, F. t. holarctica, F. t. mediasiatica, F. novicida, F. hispaniensis, F. philomiragia, and $F$. endociliophora at a temperature of $22^{\circ} \mathrm{C}$ with an infectious dose of $10^{6}$ bacteria/mL. Experiments were performed in three separate runs with ten larvae in each run for each treatment. Censored observations are indicated with a plus sign (+).

and F. hispaniensis, by $10^{4}$ over 5 days in samples infected with F. t. holarctica, and $10^{2}$ over 2 days in samples infected with $F$. philomiragia. No increase in the bacterial load of F. endociliophora in larval hemolymph was observed during the 9-day experiment (data not shown).

\section{Differences in Growth Rate Between Francisella Strains on Laboratory Culture Media}

Growth of F. hispaniensis and F. philomiragia was observed after $18 \mathrm{~h}$ incubation on solid agar media (Supplementary Figure 1). At $32 \mathrm{~h}$ colonies of the F. t. tularensis, the F. $t$. mediasiatica and the F. novicida strains were visible. However, colonies of $F$. t. holarctica and F. t. holarctica LVS were not visible until the $48 \mathrm{~h}$ time point. F. endociliophora was not included in the growth experiment since the strain does not grow at $37^{\circ} \mathrm{C}$.

\section{Host Specificity of Francisella Species}

The relevance of G. mellonella as a model system to differentiate human pathogenic strains from closely related opportunistic and non-pathogenic strains within the Francisella genus was evaluated by comparing lethality in G. mellonella (this study) with previously published data describing Francisella lethality in mice and severity of disease in humans (previously published studies and epidemiological data, respectively, Figure 4). The lethality of different Francisella strains in G. mellonella (this study) and mice were ranked as either high lethality (red), intermediate lethality (orange) and non-lethal (green). Similarly, the severity of disease caused by Francisella strains in humans was scored as incapacitating disease in humans (red), causing disease only in humans with a compromised immune response or underlying health defects (yellow), and non-virulent (green) (Figure 4). Our data show an inverse relationship, as the opportunistic pathogens in humans (F. novicida, F. hispaniensis, and F. philomiragia) are highly lethal in G. mellonella (Figure 4), while the human pathogens (F.t. tularensis and F. t. holarctica) show intermediate lethality in G. mellonella. F. endociliophora does not cause disease in either humans or G. mellonella.

\section{DISCUSSION}

Our G. mellonella model identified differences in lethality between Francisella species that do not correspond to severity of disease in humans as shown by available epidemiological and experimental data. The F. novicida, F. hispaniensis and $F$. philomiragia strains were significantly more lethal in the $G$. mellonella model than the F. $t$. holarctica and F. $t$. tularensis strains that represent human pathogens. The results provide insight into the potential host specificity of $F$. tularensis and reflect different adaptation to an insect host (G. mellonella) between $F$. tularensis and its near-neigbours. Arthropode borne transmission of human pathogenic $F$. tularensis species is widely documented and vectors considered significant for the transmission of $F$. tularensis to humans are hard tick, mosquito, deer-fly, and horse-fly (Petersen et al., 2009; Pilo, 2018). Our findings suggest that although phylogenetically close to the human pathogenic strains, the $F$. novicida, F. hispaniensis, and $F$. philomiragia are more adapted for rapid growth in the insect host model.

The strains investigated in this study were separated into three lineages (Figure 4), i.e., the F. tularensis lineage (F. t. tularensis, $F$. t. mediasiatica, F. t. holarctica, and F. t. holarctica LVS), the more diverse group comprising animal and opportunistic human pathogens associated with water environments ( $F$. novicida, $F$. hispaniensis, and F. philomiragia) and a lineage containing only $F$. endociliophora, a recently identified endosymbiont of the marine ciliate Euplotes raikovi. We can only provide speculative explanations for the inverse result of the first and second Francisella lineages in humans and G. mellonella as knowledge of Francisella spp. virulence mechanisms has mainly been restricted 


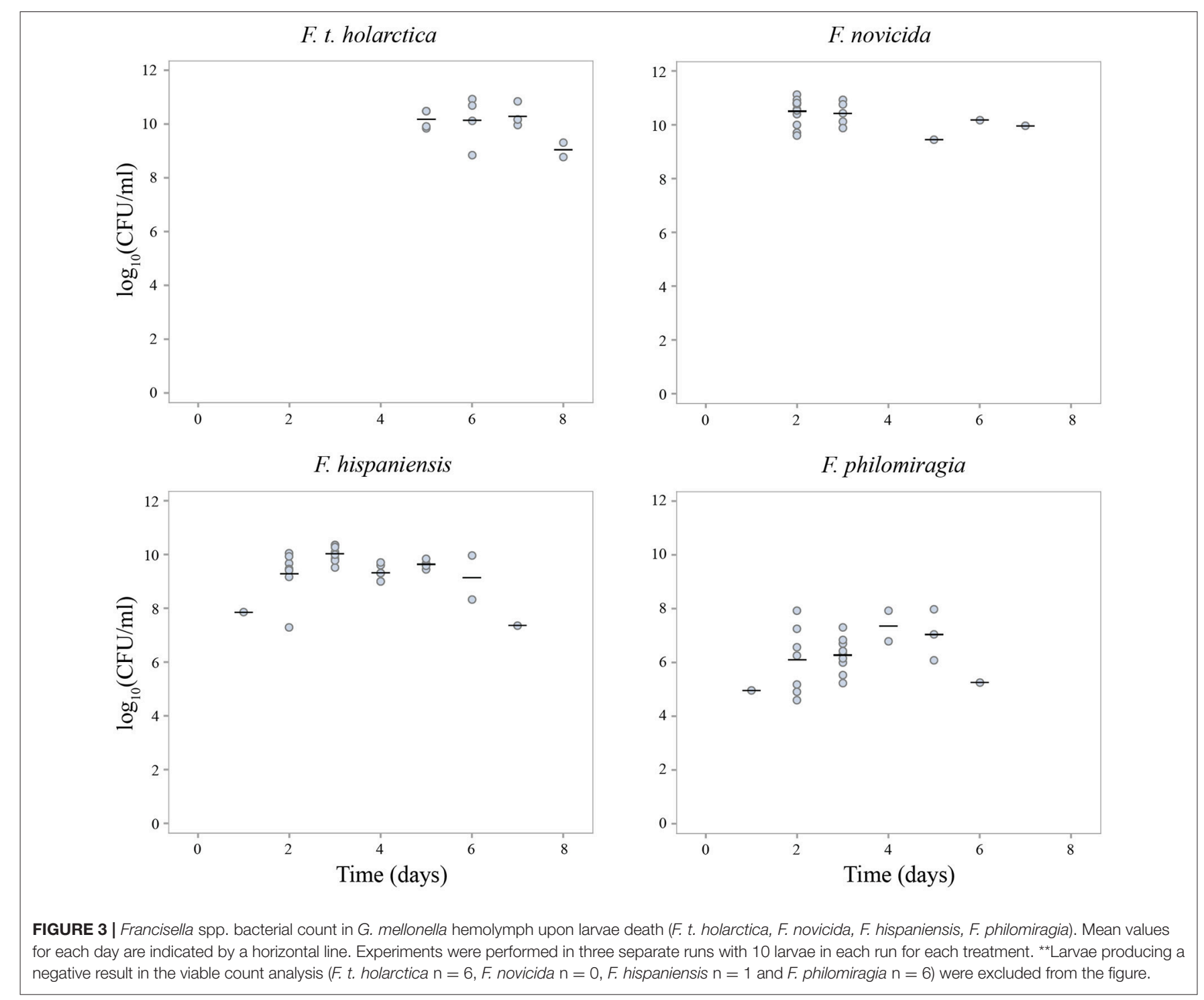

to studies of F. $t$. holarctica, F. $t$. tularensis and F. novicida. Strains in the first lineage ( $F$. tularensis), are characterized by selective genome reduction, which has resulted in deletions of metabolic pathway components (i.e., genes involved in amino acid biosynthesis) as a specialized intracellular parasite can acquire the nutrients required for growth and replication upon infecting a host (Larsson et al., 2005, 2009). In comparison, the second lineage of opportunistic strains and specifically, the extensively studied species $F$. novicida, are more metabolically versatile, less fastidious and show higher growth rates (Owen et al., 1964; Rohmer et al., 2007). In addition F. novicida elicits a different immune response in the mammalian host than F. t. tularensis and F. t. holarctica, causing disease only in immunocompromised persons (Kingry and Petersen, 2014). Our results corroborate previous studies that have shown vigorous growth of F. novicida in hemocytes (Santic et al., 2009). Studies of $F$. novicida within arthropod cells identified similar molecular mechanisms of pathogenesis, i.e., intracellular trafficking, as in mammalian cells (Ozanic et al., 2015), but the species nevertheless utilizes different virulence factors for proliferation in mammal and arthropod cells (Read et al., 2008; Santic et al., 2009; Åhlund et al., 2010).

A distinguishing feature of $F$. tularensis is its ability to evade the host immune response in mammals (Sjöstedt, 2006). F. tularensis maintain a low immunological profile early during the infection process by evading immune system surveillance, and only later replicate within the relatively protective environment of the host cell cytoplasm (Jones et al., 2014; Steiner et al., 2014). One important factor in F. tularensis immune evasion is an atypical lipopolysaccharide (LPS) that exhibits very low endotoxicity and stimulation of inflammatory pathways (Hajjar et al., 2006). In contrast, F. novicida exhibits a structurally and antigenically different LPS which elicits an inflammatory response (Jones et al., 2014). Since the growth of Francisella in hemocytes and macrophages shares many similarities, it is possible that the pathways of the G. mellonella 


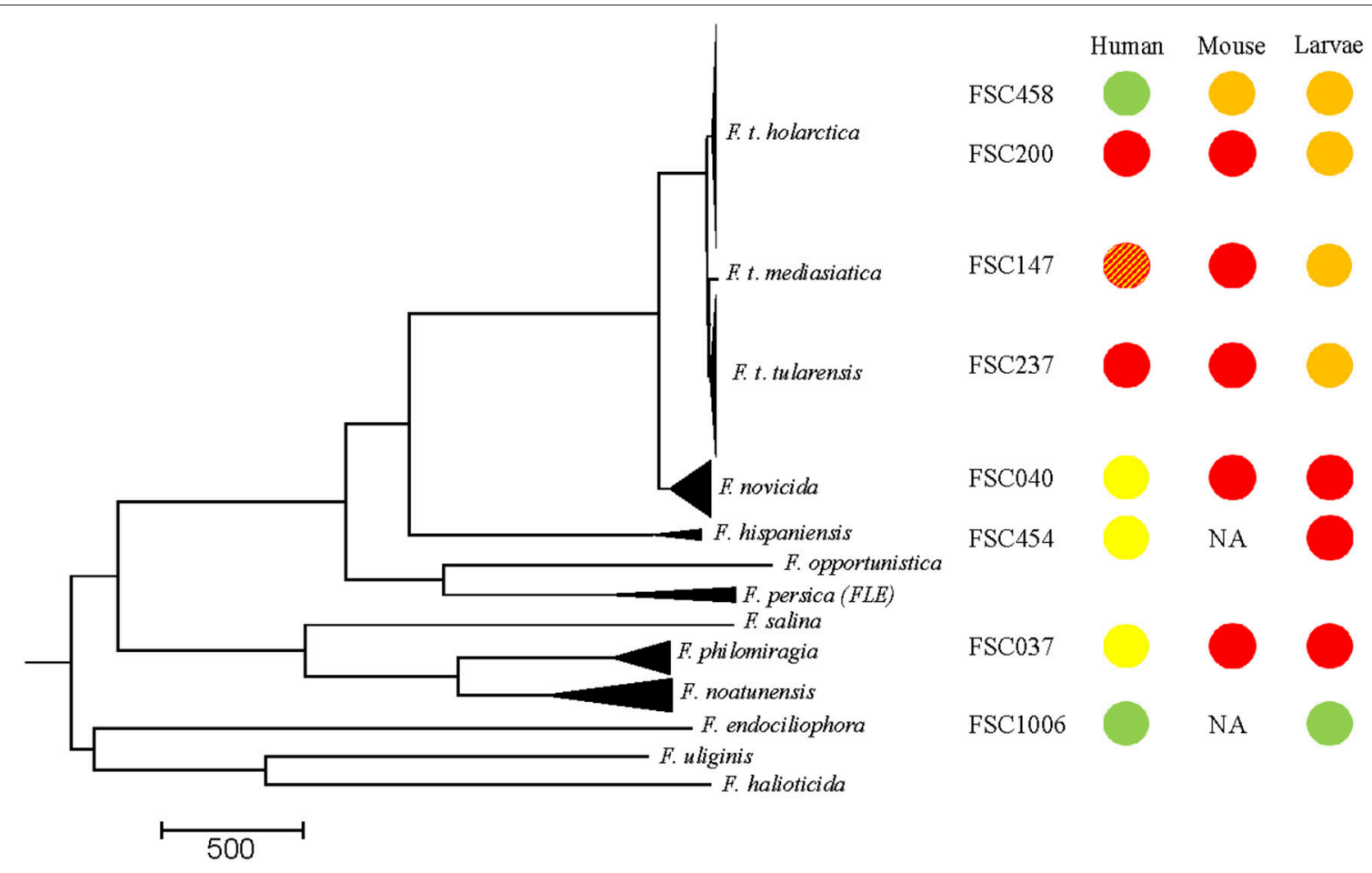

FIGURE 4 | Comparison between data of Francisella spp. lethality in G. mellonella generated in this study and previously published data on Francisella spp. lethality in mice by subcutaneous or intracutaneous inoculation (Bell et al., 1955; Owen et al., 1964; Guryčová, 1998; Kieffer et al., 2003; Salomonsson et al., 2009; Molins et al., 2014; Propst et al., 2016; Timofeev et al., 2017) and severity of disease in humans (Saslaw et al., 1961a,b; Dienst, 1963; Kugeler et al., 2009). Lethality of the different Francisella strains in G. mellonella (larvae death within 3 days, infectious dose $10^{6}$ bacteria/mL) and mice (LD $50<10^{3}$ ) was scored as high lethality (red), intermediate lethality (larvae death $>3$ days, $L D_{50}$ mice $>10^{3}$ ) (orange) and non-lethal (green). Severity of disease caused by the Francisella strains in humans, was scored as incapacitating disease in humans (red), causing disease only in humans with a compromised immune response or underlying health defects (yellow) and non-virulent (green). There is only limited data on the virulence of F. t. mediasiatica in humans (Timofeev et al., 2017) (striped) and data on F. hispaniensis and F. endociliophora lethality in mice are lacking. The LVS strain is a live attenuated strain that was selected for vaccination in humans but still remains unlicensed for human use (Sandström, 1994). NA, data not available.

humoral immune response (Browne et al., 2013; Tsai et al., 2016) differ from those in humans regarding early detection of Francisella species, and that the difference in lethality observed in our experiments (Figure 3) rather reflects growth rate differences (Supplementary Figure 1), with the metabolically versatile opportunistic Francisella strains having an advantage in G. mellonella.

The growth of $F$. philomiragia was most detrimental to the host (G. mellonella). The larvae succumbed at a lower bacterial load than what was observed for the other strains tested in this study. A more detrimental effect from growth of F. philomiragia, in comparison with $F$. tularensis ssp. and $F$. novicida, was also reported in the ciliate T. pyriformis (Thelaus et al., 2009). Virulence factors in F. philomiragia have been less studied, but based on the genome sequence, this species is likely to express similar proteins as F. tularensis and F. novicida (Zeytun et al., 2012). Previous studies have shown that F. philomiragia can infect and grow in macrophages, lung, and liver cells as well as in T. pyriformis and G. mellonella (Thelaus et al., 2009; Propst et al., 2016). This suggests that F. philomiragia, like F. tularensis ssp. and F. novicida, can infect and proliferate in many hosts and cell types. Although F. philomiragia stands out as the most aggressive strain in our experiments, all of the opportunistic strains resulted in G. mellonella death significantly faster than the three $F$. tularensis strains tested.

Finally, the third lineage, which includes only the endosymbiont $F$. endociliophora, represents a specific niche that is restricted to growth in a ciliate host and at lower temperature compared to the other strains tested (Sjödin et al., 2014). In contrast to the F. tularensis and opportunistic strains, no growth of $F$. endociliophora in G. mellonella was recorded. This is in line with the high degree of host restriction that is characteristic to primary endosymbionts, as well as the adaptation to host growth rates, which ensures that bacteria are transferred during host cell division (Fokin, 2004). Interestingly, F. endociliophora branch early in the phylogenetic tree of the Francisella genus (Figure 4) but display a more specialized lifestyle than the more recently branching Francisella species that are characterized by the ability to replicate in a broad range of hosts.

We only detected a significant difference in the lethality between wild-type $F$. $t$. holarctica and the live vaccine strain (LVS) at the intermediate infectious dose. No difference in lethality between these two strains was found in the 
G. mellonella system when the results were corrected for dose (Supplementary Table 1, column E). It could be speculated that the doses might have affected this result, with the highest dose too high to allow differentiation of the two strains, and the lower dose may have revealed differences if the study duration was extended. This should be considered in any further experiments.

Larval death following infection was less pronounced at $22^{\circ} \mathrm{C}$ than at $37^{\circ} \mathrm{C}$ for all of the tested strains, and the difference between the $F$. tularensis and opportunistic strains was less pronounced at $22^{\circ} \mathrm{C}$. It is possible that prolonged incubation would have revealed lethality differences between strains. However, G. mellonella larvae have been reported to survive incubation at $37^{\circ} \mathrm{C}$ but prolonged incubation at this temperature may induce heat stress that renders the larvae more sensitive to infection. Thus, it is plausible that the effect of temperature on larval survival can be explained by incubation at $37^{\circ} \mathrm{C}$ representing a temperature that is close to the upper limit for $G$. mellonella larvae.

In 2007, Aperis et al. introduced G. mellonella as a model host for studies of LVS and antibacterial agent efficacy (Aperis et al., 2007). In addition, Propst and colleagues compared different model systems, including G. mellonella to clarify the use of F. philomiragia as a model for studies of Francisella (Propst et al., 2016). These previous studies, along with our data, show that Francisella spp. strains multiply in, and eventually kill, G. mellonella larvae, which proves their utility as a model for virulence studies. This in line with numerous studies of G. mellonella as a model for bacterial virulence, the majority of which present good correspondence regarding bacterial pathogenicity in humans and G. mellonella (Sprynski et al., 2014; Tsai et al., 2016). Our data clearly show that the strains that cause incapacitating disease in humans are not as efficient in replicating in the larval host model as the F. novicida, F. hispaniensis, and F. philomiragia strains. Thus, Francisella spp. lethality in G. mellonella is not reflective of severity of disease in humans. This in line with a study of pathogenic Escherichia coli (causing urosepsis) in G. mellonella that conclude that the larval lethality model cannot be a substitute for the murine sepsis model (Johnson et al., 2015). On the other hand, Mukherjee et al. report on G. mellonella as a model system that reflects severity of disease of human pathogenic and non pathogenic environmental strains of Listeria spp. (Mukherjee et al., 2010). In addition, pathogenic Yersinia enterocolitica cause lethality in G. mellonella but other environmental strains of Yersinia showed high variability in insecticidal potential (Fuchs et al., 2008). Although, the innate immune responses of insects and vertebrates share a cellular and a humoral component, the evolutionary distance between insect and murine models makes it clear that many host-specific phenomena are likely to exist (Scully and Bidochka, 2006). The G. mellonella model may or may not result in a bias toward genes relevant for evading insect immunity. This will ultimately depend on the evolutionary history and ecological niche of the bacterial pathogen studied. A possible adaptation of the bacteria to an insect host would be reflected in the G. mellonella model system.

A limitation of $G$. mellonella as a model is the lack of standardization that results in unknown variation between sources that may interfere with the experimental outcome. This variation limit the possibility to directly compare data from studies performed at different laboratories. The data presented here were generated using three different batches of $G$. mellonella from the same source (Vivaria). In addition, we performed control experiments with two strains (F. t. holarctica LVS and F. novicida) using larvae from an alternative source (TruLarv ${ }^{\mathrm{TM}}$ BioSystems Technology Ltd, UK). Infection of larvae from both sources with the two strains produced similar lethality results, but larval death in the TruLarv ${ }^{\mathrm{TM}}$ system occurred 2 days later than in the Vivaria system (data not shown). This result highlights the need for comparative studies in the search for consistent model systems.

The G. mellonella data presented here illustrate possible niche differences of Francisella species and contribute to the effort to understand pathogenic F. tularensis ssp. in the context of near genetic neighbors. Larvae or insects may play a role as hosts for the replication of opportunistic human pathogens often associated with environmental samples and water. Accordingly, G. mellonella is a possible in vivo model of insect immunity that can be used for studies of both opportunistic and virulent lineages of Francisella spp., that produces inverse results regarding lethality in G. mellonella and incapacitating disease in humans. Further studies are needed in order to detangle the specific mechanism that render Francisella spp. strains lethal to G. mellonella.

\section{AUTHOR CONTRIBUTIONS}

JT designed the study, performed experiments and drafted the manuscript. EL performed experiments and contributed to the design of the study. PL contributed to the design of the study and performed the statistical analyses. AS performed the phylogenetic analysis. MF contributed to the design of the study and writing of the manuscript. All authors contributed to manuscript revision, as well as read and approved the submitted version.

\section{FUNDING}

This work was supported by the Swedish Ministry of Defense (A4042).

\section{ACKNOWLEDGMENTS}

The authors would like to thank Cecilia Ylinenjärvi for her laboratory work and Dr. Joann L Prior and her team at the Defence Science and Technology Laboratory (Dstl, United Kingdom) for introduction to the laboratory model of Galleria mellonella.

\section{SUPPLEMENTARY MATERIAL}

The Supplementary Material for this article can be found online at: https://www.frontiersin.org/articles/10.3389/fcimb. 2018.00188/full\#supplementary-material

Supplementary Figure 1 | Growt rate of Francisella spp. strains (F. t. tularensis, F. t. holarctica, F. t. mediasiatica, F. t. holarctica LVS, F. hispaniensis, F. 
philomiragia, and F. novicida) at $37^{\circ} \mathrm{C}$ on laboratory media. Bacteria was seeded onto agar plates in eight technical replicates and growth was monitored at 0, 18, 32 , and $48 \mathrm{~h}$. Growt was scored when bacterial colonies were visible to the eye.

Supplementary Table 1| $P$-values obtained from the log rank test for comparison of strains concerning survival estimates at (A) $37^{\circ} \mathrm{C}$ and

\section{REFERENCES}

Åhlund, M. K., Rydén, P., Sjöstedt, A., and Stöven, S. (2010). Directed screen of Francisella novicida virulence determinants using Drosophila melanogaster. Infect. Immun. 78, 3118-3128. doi: 10.1128/IAI.00146-10.

Aperis, G., Burgwyn Fuchs, B., Anderson, C. A., Warner, J. E., Calderwood, S. B., and Mylonakis, E. (2007). Galleria mellonella as a model host to study infection by the Francisella tularensis live vaccine strain. Microbes Infect. 9, 729-734. doi: 10.1016/j.micinf.2007.02.016

Aravena-Román, M., Merritt, A., and Inglis, T. J. J. (2015). First case of Francisella bacteraemia in Western Australia. New Microbes New Infect. 8, 75-77. doi: 10.1016/j.nmni.2015.10.004

Barnoy, S., Gancz, H., Zhu, Y., Honnold, C. L., Zurawski, D. V., and Venkatesan, M. M. (2017). The Galleria mellonella larvae as an in vivo model for evaluation of Shigella virulence. Gut Microbes 8, 335-350. doi: 10.1080/19490976.2017.1293225

Bell, J. F., Owen, C. R., and Larson, C. L. (1955). Virulence of Bacterium tularense. I. A study of the virulence of Bacterium tularense in mice, guinea pigs, and rabbits. J. Infect. Dis. 97, 162-166.

Brevik, O. J., Ottem, K. F., Kamaishi, T., Watanabe, K., and Nylund, A. (2011). Francisella halioticida sp. nov., a pathogen of farmed giant abalone (Haliotis gigantea) in Japan. J. Appl. Microbiol. 111, 1044-1056. doi: 10.1111/j.1365-2672.2011.05133.x.

Browne, N., Heelan, M., and Kavanagh, K. (2013). An analysis of the structural and functional similarities of insect hemocytes and mammalian phagocytes. Virulence 4, 597-603. doi: 10.4161/viru.25906.

Busse, H.-J., Huber, B., Anda, P., Escudero, R., Scholz, H. C., Seibold, E., et al. (2010). Objections to the transfer of Francisella novicida to the subspecies rank of Francisella tularensis - response to Johansson et al. Int. J. Syst. Evol. Microbiol. 60, 1718-1720. doi: 10.1099/00207713-60-8-1718

Challacombe, J. F., Petersen, J. M., Gallegos-Graves, L. V., Hodge, D., Pillai, S., and Kuske, C. R. (2017). Whole-genome relationships among Francisella bacteria of diverse origins define new species and provide specific regions for detection. Appl. Environ. Microbiol. 83, AEM.02589-02516. doi: 10.1128/AEM.02 589-16.

Darling, A. E., Mau, B., and Perna, N. T. (2010). ProgressiveMauve: multiple genome alignment with gene gain, loss and rearrangement. PLoS ONE 5:e11147. doi: 10.1371/journal.pone.0011147

Dienst, F. J. (1963). Tularemia - a perusal of three hundred thirty-nine cases. J. La State Med. Soc. 115, 114-127.

Federal Register (2012). Possession, Use, and Transfer of Select Agents and Toxins; Biennial Review; Final Rule. Fed. Regist. U.S. Dep. Heal. Hum. Serv. 77, 61084-61115.

Fokin, S. I. (2004). Bacterial endosymbionts of Ciliophora and their interactions with the host cell. Int. Rev. Cytol. 236, 181-249. doi: 10.1016/S0074-7696(04)36005-5.

Fuchs, T. M., Bresolin, G., Marcinowski, L., Schachtner, J., and Scherer, S. (2008). Insecticidal genes of Yersinia spp.: Taxonomical distribution, contribution to toxicity towards Manduca sexta and Galleria mellonella, and evolution. BMC Microbiol. 8:214. doi: 10.1186/1471-2180-8-214

Guryčová, D. (1998). First isolation of Francisella tularensis subsp. tularensis in Europe. Eur. J. Epidemiol. 14, 797-802. doi: 10.1023/A:1007537405242

Hajjar, A. M., Harvey, M. D., Shaffer, S. A., Goodlett, D. R., Sjöstedt, A., Edebro, H., et al. (2006). Lack of in vitro and in vivo recognition of Francisella tularensis subspecies lipopolysaccharide by Toll-like receptors. Infect. Immun. 74, 6730-6738. doi: 10.1128/IAI.00934-06

Hollis, D. G., Weaver, R. E., Steigerwalt, A. G., Wenger, J. D., Moss, W. C., and Brenner, D. J. (1989). Francisella philomiragia comb. nov. (formerly Yersinia philomiragia) and Francisella tularensis biogroup novicida (formerly Francisella novicida) associated with human disease. J. Clin. Microbiol. 27, 1601-1608. infectious dose of $10^{4}$ bacteria/mL, (B) $37^{\circ} \mathrm{C}$ and infectious dose of $10^{6}$ bacteria $/ \mathrm{mL}$, (C) $37^{\circ} \mathrm{C}$ and infectious dose of $10^{8}$ bacteria/mL, (D) $22^{\circ} \mathrm{C}$ and infectious dose of $10^{6}$ bacteria/mL and $(E) 37^{\circ} \mathrm{C}$ controlled for infectious dose. The $p$-values are adjusted for multiple testing according to the Tukey-Kramer method.

Huber, B., Escudero, R., Busse, H.-J., Seibold, E., Scholz, H. C., Anda, P., et al. (2010). Description of Francisella hispaniensis sp. nov., isolated from human blood, reclassification of Francisella novicida (Larson et al. 1955) Olsufiev et al. 1959 as Francisella tularensis subsp. novicida comb. nov. and emended description of the genus Franc. Int. J. Syst. Evol. Microbiol. 60, 1887-1896. doi: 10.1099/ijs.0.015941-0

Johansson, A., Celli, J., Conlan, W., Elkins, K. L., Forsman, M., Keim, P. S., et al. (2010). Objections to the transfer of Francisella novicida to the subspecies rank of Francisella tularensis. Int. J. Syst. Evol. Microbiol. 60, 1717-1718. doi: 10.1099/ijs.0.022830-0.

Johnson, J. R., Porter, S., Johnston, B., Kuskowski, M. A., Spurbeck, R. R., Mobley, H. L. T., et al. (2015). Host Characteristics and bacterial traits predict experimental virulence for Escherichia coli bloodstream isolates from patients with urosepsis. Open Forum Infect. Dis. 2. doi: 10.1093/ofid/ofv083

Jones, B. D., Faron, M., Rasmussen, J. A., and Fletcher, J. R. (2014). Uncovering the components of the Francisella tularensis virulence stealth strategy. Front. Cell. Infect. Microbiol. 4:32. doi: 10.3389/fcimb.2014.00032

Kavanagh, K., and Reeves, E. P. (2004). Exploiting the potential of insects for in vivo pathogenicity testing of microbial pathogens. FEMS Microbiol. Rev. 28, 101-112. doi: 10.1016/j.femsre.2003.09.002

Kieffer, T. L., Cowley, S., Nano, F. E., and Elkins, K. L. (2003). Francisella novicida LPS has greater immunobiological activity in mice than $F$. tularensis LPS, and contributes to F. novicida murine pathogenesis. Microbes Infect. 5, 397-403. doi: 10.1016/S1286-4579(03)00052-2

Kingry, L. C., and Petersen, J. M. (2014). Comparative review of Francisella tularensis and Francisella novicida. Front. Cell. Infect. Microbiol. 4:35. doi: $10.3389 /$ fcimb.2014.00035

Köster, J., and Rahmann, S. (2012). Snakemake-a scalable bioinformatics workflow engine. Bioinformatics 28, 2520-2522. doi: 10.1093/bioinformatics/bts480

Kugeler, K. J., Mead, P. S., Janusz, A. M., Staples, J. E., Kubota, K. A., Chalcraft, L. G., et al. (2009). Molecular Epidemiology of Francisella tularensis in the United States. Clin. Infect. Dis. 48, 863-870. doi: 10.1086/ 597261

Kumar, S., Stecher, G., and Tamura, K. (2016). MEGA7: molecular evolutionary genetics analysis version 7.0 for bigger datasets. Mol. Biol. Evol. 33, 1870-1874. doi: 10.1093/molbev/msw054

Lärkeryd, A., Myrtennäs, K., Karlsson, E., Dwibedi, C. K., Forsman, M., Larsson, P., et al. (2014). CanSNPer: a hierarchical genotype classifier of clonal pathogens. Bioinformatics 30, 1762-1764. doi: 10.1093/bioinformatics/btu113

Larson, C., Wicht, W., and Jellison, W. (1955). A new organism resembling P. tularensis isolated from water. Public Health Rep. 70, 253-258.

Larson, M. A., Nalbantoglu, U., Sayood, K., Zentz, E. B., Cer, R. Z., Iwen, P. C., et al. (2016). Reclassification of Wolbachia persica as Francisella persica comb. nov. and emended description of the family Francisellaceae. Int. J. Syst. Evol. Microbiol. 66, 1200-1205. doi: 10.1099/ijsem.0.000855

Larsson, P., Elfsmark, D., Svensson, K., Wikström, P., Forsman, M., Brettin, T., et al. (2009). Molecular evolutionary consequences of niche restriction in Francisella tularensis, a facultative intracellular pathogen. PLoS Pathog. 5:e1000472. doi: 10.1371/journal.ppat.1000472

Larsson, P., Oyston, P. C., Chain, P., Chu, M. C., Duffield, M., Fuxelius, H. H., et al. (2005). The complete genome sequence of Francisella tularensis, the causative agent of tularemia. Nat. Genet. 37, 153-159. doi: 10.1038/ ng1499

Meir, M., Grosfeld, T., and Barkan, D. (2018). Establishment and validation of Galleria mellonella as a novel model organism to study Mycobacterium abscessus infection, pathogenesis and treatment. Antimicrob. Agents Chemother. 62:e02539-17. doi: 10.1128/AAC.02539-17

Molins, C. R., Delorey, M. J., Yockey, B. M., Young, J. W., Belisle, J. T., Schriefer, M. E., et al. (2014). Virulence difference between the prototypic Schu S4 strain (A1a) and Francisella tularensis A1a, A1b, A2 and type B strains in 
a murine model of infection. BMC Infect. Dis. 14:67. doi: 10.1186/14712334-14-67.

Mukherjee, K., Altincicek, B., Hain, T., Domann, E., Vilcinskas, A., and Chakraborty, T. (2010). Galleria mellonella as a model system for studying Listeria pathogenesis. Appl. Environ. Microbiol. 76, 310-317. doi: 10.1128/AEM.01301-09.

Olsufjev, N. G., Emelyanova, O. S., and Dunaeva, T. N. (1959). Comparative study of strains of B. tularense in the Old andNew World and their taxonomy. Hyg. Epidemiol. Microbiol. Immunol. 3, 138-149.

Ottem, K. F., Nylund, A., Karlsbakk, E., Friis-Møller, A., and Kamaishi, T. (2008). Elevation of Francisella philomiragia subsp. noatunensis Mikalsen et al. (2007) to Francisella noatunensis comb. nov. [syn. Francisella piscicida Ottem et al. (2008) syn. nov.] and characterization of Francisella noatunensis subsp. orientalis subsp. nov. J. Appl. Microbiol. 106, 1231-1243. doi: 10.1111/j.1365-2672.2008.04092.x

Owen, C. R., Buker, E. O., Jellison, W. L., Lackman, D. B., and Bell, J. F. (1964). Comparative studies of Francisella tularensis and Francisella novicida. J. Bacteriol. 87, 676-683.

Ozanic, M., Marecic, V., Abu Kwaik, Y., and Santic, M. (2015). The divergent intracellular lifestyle of Francisella tularensis in evolutionarily distinct host cells. PLoS Pathog. 11:e1005208. doi: 10.1371/journal.ppat. 1005208

Penn, R. (2010). "Francisella tularensis (Tularemia)," in Mandell, Douglas and Bennett's Principles and Practice of Infectious Diseases, eds G. L. Mandell, J. E. Bennet, and R. Doblin (Philadelphia PA: Elsevier Churchill, Livingstone), 2927-2937.

Petersen, J. M., Mead, P. S., and Schriefer, M. E. (2009). Francisella tularensis : an arthropod-borne pathogen. Vet. Res. 40:7. doi: 10.1051/vetres:2008045

Pilo, P. (2018). Arthropod Infection Models for Francisella tularensis. Curr. Clin. Microbiol. Reports 5, 10-17. doi: 10.1007/s40588-018-0084-Z

Propst, C. N., Pylypko, S. L., Blower, R. J., Ahmad, S., Mansoor, M., and van Hoek, M. L. (2016). Francisella philomiragia Infection and Lethality in Mammalian Tissue Culture Cell Models, Galleria mellonella, and BALB/c Mice. Front. Microbiol. 7:696. doi: 10.3389/fmicb.2016.00696

Qu, P.-H., Chen, S.-Y., Scholz, H. C., Busse, H.-J., Gu, Q., Kämpfer, P., et al. (2013). Francisella guangzhouensis sp. nov., isolated from air-conditioning systems. Int. J. Syst. Evol. Microbiol. 63, 3628-3635. doi: 10.1099/ijs.0.049916-0

Qu, P.-H., Li, Y., Salam, N., Chen, S.-Y., Liu, L., Gu, Q., et al. (2016). Allofrancisella inopinata gen. nov., sp. nov. and Allofrancisella frigidaquae sp. nov., isolated from water-cooling systems, and transfer of Francisella guangzhouensis Qu et al. 2013 to the new genus as Allofrancisella guangzhouensis comb. nov. Int. J. Syst. Evol. Microbiol. 66, 4832-4838. doi: 10.1099/ijsem.0.001437

Read, A., Vogl, S. J., Hueffer, K., Gallagher, L. A., and Happ, G. M. (2008). Francisella genes required for replication in mosquito cells. J. Med. Entomol. 45, 1108-1116. doi: 10.1603/0022-2585(2008)451108:FGRFRI2.0.CO;2

Rohmer, L., Fong, C., Abmayr, S., Wasnick, M., Larson Freeman, T. J., Radey, M., et al. (2007). Comparison of Francisella tularensis genomes reveals evolutionary events associated with the emergence of human pathogenic strains. Genome Biol. 8:R102. doi: 10.1186/gb-2007-8-6-r102

Salomonsson, E., Kuoppa, K., Forslund, A. L., Zingmark, C., Golovliov, I., Sjöstedt, A., et al. (2009). Reintroduction of two deleted virulence loci restores full virulence to the live vaccine strain of Francisella tularensis. Infect. Immun. 77, 3424-3431. doi: 10.1128/IAI.00196-09

Sandström, G. (1994). The tularaemia vaccine. J. Chem. Technol. Biotechnol. 59, 315-320. doi: 10.1002/jctb.280590402

Santic, M., Akimana, C., Asare, R., Kouokam, J. C., Atay, S., and Kwaik, Y. A. (2009). Intracellular fate of Francisella tularensis within arthropod-derived cells. Environ. Microbiol. 11, 1473-1481. doi: 10.1111/j.1462-2920.2009.01875.x

Saslaw, S., Eigelsbach, H. T., Prior, J. A., Wilson, H. E., and Carhart, S. (1961a). Tularemia vaccine study. I. Intracutaneous challenge. Arch. Intern. Med. 107, 689-701. doi: 10.1001/archinte.1961.03620050055006
Saslaw, S., Eigelsbach, H. T., Prior, J. A., Wilson, H. E., and Carhart, S. (1961b). Tularemia vaccine study. II. Respiratory challenge. Arch. Intern. Med. 107, 702-714.

Scully, L. R., and Bidochka, M. J. (2006). Developing insect models for the study of current and emerging human pathogens. FEMS Microbiol. Lett. 263, 1-9. doi: 10.1111/j.1574-6968.2006.00388.x

Sjödin, A., Öhrman, C., Bäckman, S., Lärkeryd, A., and Granberg, M., Lundmark, E., et al. (2014). Complete genome sequence of Francisella endociliophora strain FSC1006, isolated from a laboratory culture of the marine ciliate Euplotes raikovi. Genome Announc. 2, 6-7. doi: 10.1128/genomeA.01227-14. Copyright

Sjödin, A., Svensson, K., Öhrman, C., Ahlinder, J., Lindgren, P., Duodu, S., et al. (2012). Genome characterisation of the genus Francisella reveals insight into similar evolutionary paths in pathogens of mammals and fish. BMC Genomics 13:268. doi: 10.1186/1471-2164-13-268

Sjöstedt, A. (2006). Intracellular survival mechanisms of Francisella tularensis, a stealth pathogen. Microbes Infect. 8, 561-567. doi: 10.1016/j.micinf.2005.08.001

Sjöstedt, A. B. (2015). “Francisellaceae fam. nov.", in Bergey's Manual of Systematics of Archaea and Bacteria, eds G. Garrity, J. Don, N. Krieg, and J. Staley (Chichester: John Wiley \& Sons, Ltd), 1-2.

Sprynski, N., Valade, E., and Neulat-Ripoll, F. (2014). Galleria mellonella as an Infection Model for Select Agents. New York, NY: Humana Press.

Steiner, D. J., Furuya, Y., and Metzger, D. (2014). Host-pathogen interactions and immune evasion strategies in Francisella tularensis pathogenicity. Infect. Drug Resist. 7, 239-251. doi: 10.2147/IDR.S53700

Svensson, K., Sjodin, A., Bystrom, M., Granberg, M., Brittnacher, M. J., Rohmer, L., et al. (2012). Genome Sequence of Francisella tularensis subspecies holarctica Strain FSC200, Isolated from a Child with Tularemia. J. Bacteriol. 194, 6965-6966. doi: 10.1128/JB.01040-12

Thelaus, J., Andersson, A., Mathisen, P., Forslund, A.-L., Noppa, L., and Forsman, M. (2009). Influence of nutrient status and grazing pressure on the fate of Francisella tularensis in lake water. FEMS Microbiol. Ecol. 67, 69-80. doi: 10.1111/j.1574-6941.2008.00612.x

Timofeev, V., Bakhteeva, I., Titareva, G., Kopylov, P., Christiany, D., Mokrievich, A., et al. (2017). Russian isolates enlarge the known geographic diversity of Francisella tularensis subsp. mediasiatica. PLoS ONE 12:e0183714. doi: 10.1371/journal.pone.0183714

Tsai, C. J.-Y., Loh, J. M. S., and Proft, T. (2016). Galleria mellonella infection models for the study of bacterial diseases and for antimicrobial drug testing. Virulence 7, 214-229. doi: 10.1080/21505594.2015.1135289

Versage, J. L., Severin, D. D., Chu, M. C., and Petersen, J. M. (2003). Development of a multitarget real-time TaqMan PCR assay for enhanced detection of Francisella tularensis in complex specimens. J. Clin. Microbiol. 41, 5492-5499. doi: 10.1128/JCM.41.12.5492-5499.2003

World Health Organization, WHO (2007). WHO Guidelines on Tularaemia. Geneva: WHO Press.

Zeytun, A., Malfatti, S. A., Vergez, L. M., Shin, M., Garcia, E., and Chain, P. S. G. (2012). Complete genome sequence of Francisella philomiragia ATCC 25017. J. Bacteriol. 194, 3266-3265. doi: 10.1128/JB.00413-12

Conflict of Interest Statement: The authors declare that the research was conducted in the absence of any commercial or financial relationships that could be construed as a potential conflict of interest.

Copyright (C) 2018 Thelaus, Lundmark, Lindgren, Sjödin and Forsman. This is an open-access article distributed under the terms of the Creative Commons Attribution License (CC BY). The use, distribution or reproduction in other forums is permitted, provided the original author(s) and the copyright owner are credited and that the original publication in this journal is cited, in accordance with accepted academic practice. No use, distribution or reproduction is permitted which does not comply with these terms. 